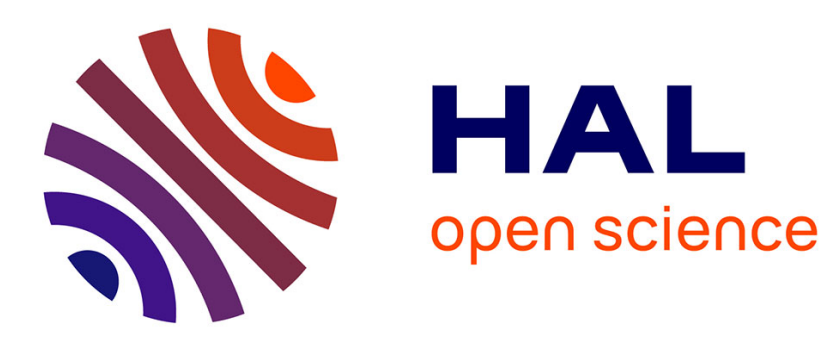

\title{
GALERKIN METHOD FOR TIME FRACTIONAL SEMILINEAR EQUATIONS
}

Yamina Ouedjedi, Arnaud Rougirel, Khaled Benmeriem

\section{To cite this version:}

Yamina Ouedjedi, Arnaud Rougirel, Khaled Benmeriem. GALERKIN METHOD FOR TIME FRACTIONAL SEMILINEAR EQUATIONS. 2019. hal-02124150

\section{HAL Id: hal-02124150 \\ https://hal.science/hal-02124150}

Preprint submitted on 9 May 2019

HAL is a multi-disciplinary open access archive for the deposit and dissemination of scientific research documents, whether they are published or not. The documents may come from teaching and research institutions in France or abroad, or from public or private research centers.
L'archive ouverte pluridisciplinaire HAL, est destinée au dépôt et à la diffusion de documents scientifiques de niveau recherche, publiés ou non, émanant des établissements d'enseignement et de recherche français ou étrangers, des laboratoires publics ou privés. 


\title{
GALERKIN METHOD FOR TIME FRACTIONAL SEMILINEAR EQUATIONS
}

\author{
YAMINA OUEDJEDI, ARNAUD ROUGIREL, KHALED BENMERIEM
}

\begin{abstract}
This paper gathers the tools for solving Riemann-Liouville time fractional non-linear reaction-diffusion equations by the Galerkin method. As a model problem, existence and uniqueness is proved for semilinear heat equations with polynomial growth at infinity.
\end{abstract}

\section{INTRODUCTION}

The Galerkin approximation method in an efficient and robust tool for solving partial differential equations (see for instance [Tem88], [Lio69], Rou05]). In that paper, only low order Riemann-Liouville time fractional derivatives are considered; that is to say, the order of the fractional derivatives is less than 1. In order to implement this method for solving time fractional non-linear problems, three tools have to be developed: (i) a suitable functional framework; (ii) time fractional inequalities; (iii) an Aubin-Lions theory. Let us introduce these elements.

Usually, fractional Gagliardo-Sobolev spaces are used. However, they are not very suitable for time fractional problems since the connection between these spaces and time fractional derivatives is not straightforward. The consequence is that a trivial initial condition is needed. Moreover, these spaces are quite complicated to handle.

Recently, suitable and simpler fractional spaces appear in the literature. See for instance [DR18], LL18b], dF19]. These spaces are natural generalizations of the spaces involved in the integer case. See Section 3 for details.

By time fractional inequalities, we means for example

$$
\frac{1}{2} \mathrm{D}_{0, t}^{\alpha} \int_{\Omega} u(t, x)^{2} \mathrm{~d} x \leq \int_{\Omega} \mathrm{D}_{0, t}^{\alpha} u(t, x) u(t, x) \mathrm{d} x .
$$

According to the integer setting, we cannot expect such an relationship to hold without imposing a zero initial condition. Under some smoothness conditions, (1.1) is proved in [Zac12, Theorem 2.4], and in [LL18b, Proposition 2.18] by a simple and smart convexity argument. In order to apply (1.1) to nonlinear problems, we have relaxed the smoothness assumptions by a density argument: see Corollary 3.3 and Proposition 3.4 where a integral version of (1.1) is featured. We refer also to dF19], where time fractional inequalities hold for hilbert valued functions. However,

Aubin-Lions theory allows to get point-wise convergence by compactness arguments, and to pass to the limit in non-linear terms. By adapting the arguments of [LL18b], we obtain the compactness result stated in Corollary 3.5 .

Key words and phrases. Riemann-Liouville derivatives, Galerkin method, Fractional semilinear equations.

May 2, 2019. 
In the two forcoming sections, we recall or develop the tools for solving fractional equations. The Galerkin method is implemented in section 4 for solving time fractional semilinear heat equations.

\section{Preliminaries}

As far as integrable functions are concerned, convolution is a basic tool in fractional calculus. However, in order to obtain a density result, namely Theorem 3.1, we will need to make the convolution of non absolutely integrable functions. That can be achieved following [GS77], for causal functions.

Let $(X,\|\|$.$) be a real Banach space, and T$ be a positive number. Let us recall that $f \in L_{\mathrm{loc}}^{1}(\mathbb{R} ; X)$ is said to be causal if $f=0$ a.e. on $(-\infty, 0)$.

Definition 2.1. Let $f \in L_{\text {loc }}^{1}(\mathbb{R} ; X), g \in L_{\text {loc }}^{1}(\mathbb{R})$ be causal functions. Then the convolution of $f$ and $g$ is the causal function of $L_{\text {loc }}^{1}(\mathbb{R} ; X)$ defined, for a.e. $t \in \mathbb{R}$, by

$$
g * f(t)=\int_{\mathbb{R}} g(t-y) f(y) \mathrm{d} y .
$$

Classically, fractional derivatives involve another kind of convolution, since the functions are defined on $[0, T]$.

Definition 2.2. Let $f \in L^{1}(0, T ; X)$ and $g \in L^{1}(0, T)$. Then the convolution of $g$ and $f$ is the element of $L^{1}(0, T ; X)$ defined, for a.e. $t \in[0, T]$, by

$$
g *_{T} f(t):=\int_{0}^{t} g(t-y) f(y) \mathrm{d} y .
$$

Of course these two definitions are consistent. Indeed, the following result hods true.

Proposition 2.1. Let $f \in L_{\mathrm{loc}}^{1}(\mathbb{R} ; X)$ and $g \in L_{\mathrm{loc}}^{1}(\mathbb{R})$ be causal functions. Then

$$
g_{\left.\right|_{[0, T]}} *_{T} f_{\left.\right|_{[0, T]}}=(g * f)_{\left.\right|_{[0, T]}} \quad \text { in } \quad L^{1}(0, T ; X) .
$$

Above, $f_{\mid[0, T]}$ denotes the restriction of $f$ to $[0, T]$. The elementary proof of that proposition is omitted. Owing to the above result, we will write $g * f$ instead of $g *_{T} f$, if no confusion can occur.

The following standard inequality will be useful. Let $I=[0, T]$ or $\mathbb{R}$. If $f \in L^{p}(I ; X)$ with $1 \leq p \leq \infty$, and $g \in L^{1}(I)$ then $g * f$ belongs to $L^{p}(I ; X)$ and

$$
\|g * f\|_{L^{p}(I ; X)} \leq\|g\|_{L^{1}(I)}\|f\|_{L^{p}(I ; X)} .
$$

Let us turn our attention to fractional derivatives. The following kernels are of fundamental importance in the theory of fractional derivatives. For $\alpha \in(0, \infty)$, we denote by $g_{\alpha}$ the causal function of $L_{\mathrm{loc}}^{1}(\mathbb{R})$ defined, for a.e. $t>0$, by

$$
g_{\alpha}(t)=\frac{1}{\Gamma(\alpha)} t^{\alpha-1}
$$

These Kernels satisfy the following semi-group property : for $\alpha>0, \beta>0$,

$$
g_{\alpha} * g_{\beta}=g_{\alpha+\beta} \quad \text { in } L_{\mathrm{loc}}^{1}(\mathbb{R}) .
$$

Now, we are able to introduce the fractional Riemann-Liouville derivative of vectorvalued functions. In the sequel, $\alpha \in(0,1)$ will denote the fractional order of differentiation. 
Definition 2.3. Let $1 \leq q<\infty$ and $u \in L^{q}(0, T ; X)$. We say that $u$ admits a fractional derivative of order $\alpha$ in $L^{q}(0, T ; X)$ if

$$
g_{1-\alpha} * u \in W^{1, q}(0, T ; X) .
$$

In this case, the fractional derivative of order $\alpha$ of $u$ is the function of $L^{q}(0, T ; X)$ defined by

$$
\mathrm{D}_{0, t}^{\alpha} u:=\frac{\mathrm{d}}{\mathrm{d} t}\left\{g_{1-\alpha} * u\right\} .
$$

Above, $W^{1, q}(0, T ; X)$ denotes the space of functions belonging to $L^{q}(0, T ; X)$ whose first order derivative (in the sense of distributions) belongs to $L^{q}(0, T ; X)$.

Proposition 2.2. Let $1 \leq q<\infty, \alpha \in(0,1)$ and $u \in L^{q}(0, T ; X)$. If $u$ admits a fractional derivative in $L^{q}(0, T ; X)$ then

$$
u=\left(g_{1-\alpha} * u\right)(0) g_{\alpha}+g_{\alpha} * \mathrm{D}_{0, t}^{\alpha} u \quad \text { in } L^{q}(0, T ; X) .
$$

Moreover, if $\alpha \leq 1-\frac{1}{q}$ then $\left(g_{1-\alpha} * u\right)(0)=0$.

Any function $u$ satisfying the assumptions of Proposition 2.2 has, in some sense, a weak singularity at $t=0$. Indeed, let us assume that $u=v g_{\beta}$ where $v \in X \backslash\{0\}$ and $\beta>0$. Then by 2.2 , $u$ belongs to $L^{q}(0, T ; X)$ and has a $\alpha$-derivative in $L^{q}(0, T ; X)$ iff $\beta>1-\frac{1}{q}$ or $\beta=\alpha$.

Proof of Proposition 2.2. Equality (2.3) is well known (see for instance [DR18, Proposition $3.4])$. In order to prove the second assertion, we observe that $g_{\alpha}$ does not belong to $L^{q}(0, T)$ if $\alpha \leq 1-\frac{1}{q}$. Thus we must have $\left(g_{1-\alpha} * u\right)(0)=0$.

Let us now focus on weak fractional derivative of vector-valued functions. The starting point is the following standard integration by-part formula.

Proposition 2.3. [DR18, Proposition 3.1] Let $\alpha \in(0,1), f \in L^{1}(0, T ; X)$ and $\psi \in$ $C^{1}([0, T])$. Assume that $f$ admits a derivative of order $\alpha$ in $L^{1}(0, T ; X)$. Then

$$
\int_{0}^{T} \mathrm{D}_{0, t}^{\alpha} f(t) \psi(t) \mathrm{d} t=-\int_{0}^{T} f(t) \mathrm{D}_{t, T}^{\alpha} \psi(t) \mathrm{d} t+\left[g_{1-\alpha} * f \psi\right]_{0}^{T} \quad \text { in } \quad X,
$$

where

$$
\mathrm{D}_{t, T}^{\alpha} \psi(t):=\int_{t}^{T} g_{1-\alpha}(y-t) \psi^{\prime}(y) \mathrm{d} y, \quad \forall t \in[0, T],
$$

and $\psi^{\prime}:=\frac{\mathrm{d}}{\mathrm{d} t} \psi$ denotes the derivative of $\psi$. Moreover, if in addition, $\psi(0)=\psi(T)=0$ then

$$
\left\|\int_{0}^{T} f(t) D_{t, T}^{\alpha} \psi(t) \mathrm{d} t\right\| \leq g_{2-\alpha}(T)\|f\|_{L^{q}(0, T ; X)}\left\|\psi^{\prime}\right\|_{L^{\infty}(0, T)} .
$$

That property allows us to define fractional derivative in the sense of distributions. Indeed, 2.5 shows that the linear map

$$
\mathcal{D}(0, T) \rightarrow X, \quad \varphi \mapsto-\int_{0}^{T} f(t) \mathrm{D}_{t, T}^{\alpha} \varphi(t) \mathrm{d} t
$$

is a vector-valued distribution, whose order is (at most) 1 . The set of distributions with values in $X$ is denoted by $\mathcal{D}^{\prime}(0, T ; X)$. That allows us to set this definition. 
Definition 2.4. Let $\alpha \in(0,1), q \in[1, \infty)$ and $f \in L^{q}(0, T ; X)$. Then the weak derivative of order $\alpha$ of $f$ is the vector-valued distribution, denoted by $\mathrm{D}_{0, t}^{\alpha} f$, and defined, for all $\varphi \in \mathcal{D}(0, T)$, by

$$
\left\langle\mathrm{D}_{0, t}^{\alpha} f, \varphi\right\rangle=-\int_{0}^{T} f(t) \mathrm{D}_{t, T}^{\alpha} \varphi(t) \mathrm{d} t \quad \text { in } X
$$

If we want to highlight the duality taking place in the above bracket, we will write

$$
\left\langle\mathrm{D}_{0, t}^{\alpha} f, \varphi\right\rangle_{\mathcal{D}^{\prime}(0, T ; X), \mathcal{D}(0, T)}
$$

instead of $\left\langle\mathrm{D}_{0, t}^{\alpha} f, \varphi\right\rangle$.

Clearly, weak fractional derivatives is a natural extension of (first order) weak derivatives, also called derivatives in the sense of distribution.

Of course, the concept of weak derivative extends that of fractional derivative given in Definition 2.3. See [DR18, Proposition 3.2] for details. Finally, we recall a proposition useful for passing to the limit in fractional derivatives.

Proposition 2.4. [DR18, Proposition 3.3] Let $\alpha \in(0,1), V$ be a real Banach space, $q \in[1, \infty)$, and $f \in L^{q}\left(0, T ; V^{\prime}\right)$. We assume that $f$ admits a derivative of order $\alpha$ in $L^{q}\left(0, T ; V^{\prime}\right)$. Then, for each $v$ in $V,\langle f, v\rangle_{V^{\prime}, V}$ admits a derivative of order $\alpha$ in $L^{q}(0, T)$ and

$$
\left\langle\mathrm{D}_{0, t}^{\alpha} f(\cdot), v\right\rangle_{V^{\prime}, V}=\mathrm{D}_{0, t}^{\alpha}\left\{\langle f, v\rangle_{V^{\prime}, V}\right\}, \quad \text { in } L^{q}(0, T) .
$$

Here, $V^{\prime}$ denotes the dual space of $V$ and $\langle\cdot, \cdot\rangle_{V^{\prime}, V}$ the corresponding duality bracket.

\section{FRACTIONAL SPACES}

In this section, we introduce the functional framework for solving fractional semilinear equations. Let $X, Y$ be real Banach spaces such that $X$ is continuously embedded into $Y$. Also, let $T>0, \alpha \in(0,1)$ and $p, q \in[1, \infty)$. Then we introduce the following space

$$
W_{p, q}^{\alpha}(0, T ; X, Y):=\left\{u \in L^{p}(0, T ; X): \mathrm{D}_{0, t}^{\alpha} u \in L^{q}(0, T ; Y)\right\}
$$

and

$$
{ }_{0} W_{p, q}^{\alpha}(0, T ; X, Y):=\left\{u \in W_{p, q}^{\alpha}(0, T ; X, Y):\left(g_{1-\alpha} * u\right)(0)=0 \text { in } Y\right\} .
$$

$W_{p, q}^{1}(0, T ; X, Y)$ is the standard Sobolev space used for solving non-linear PDE's by the Galerkin method (see for instance [Rou05]). Therefore, $W_{p, q}^{\alpha}(0, T ; X, Y)$ is the "simplest" spaces we can think of when solving (4.1).

In (3.1), $\mathrm{D}_{0, t}^{\alpha} u$ is understood in the sense of distribution, i.e. in the sense of Definition 2.4. Alternatively, $W_{p, q}^{\alpha}(0, T ; X, Y)$ may be defined trough Definition 2.3, as the set of functions in $L^{p}(0, T ; X)$ which admits a fractional derivative in $L^{q}(0, T ; Y)$.

Equipped with the norm

$$
\|u\|_{W^{\alpha}}:=\left(\|u\|_{L^{p}(0, T ; X)}^{2}+\left\|\mathrm{D}_{0, t}^{\alpha} u\right\|_{L^{q}(0, T ; Y)}^{2}\right)^{1 / 2},
$$

it is clear that $W_{p, q}^{\alpha}(0, T ; X, Y)$ and ${ }_{0} W_{p, q}^{\alpha}(0, T ; X, Y)$, are Banach spaces.

We start by a density result. As far as the above fractional spaces are considered, such results are quite uncommon in the literature (see however [dF19, Theorem 39]). This Theorem allows to extend the coercivity result of [LL18b] from an hilbertian setting into Banach setting. 
Theorem 3.1. Let $X, Y$ be real Banach spaces such that $X$ is continuously embedded into $Y$ and, for $p, q \in[1, \infty)$,

$$
u \in{ }_{0} W_{p, q}^{\alpha}(0, T ; X, Y) .
$$

Then there exists a sequence $\left(u_{n}\right)_{n \geq 1}$ in $C^{\infty}([0, T] ; X)$ such that $u_{n}(0)=0$ for each $n$, and

$$
u_{n} \rightarrow u, \quad \text { in }{ }_{0} W_{p, q}^{\alpha}(0, T ; X, Y) \text {. }
$$

To prove this theorem we use the following lemma, whose proof can be found in Oue.

Lemma 3.2. Let $T>0$ and $u \in L_{\mathrm{loc}}^{1}(\mathbb{R} ; X)$ be a causal function such that $u_{[0, T]}$ belongs ${ }_{0} W_{p, q}^{\alpha}(0, T ; X, Y)$. Then, for each $h>0, u(\cdot-h)$ lies in ${ }_{0} W_{p, q}^{\alpha}(0, T ; X, Y)$ and

$$
u(\cdot-h) \underset{h \rightarrow 0}{\longrightarrow} u, \quad \text { in } W_{p, q}^{\alpha}(0, T ; X, Y) .
$$

Proof of Theorem 3.1. By lemma 3.2 we may assume that there exists $h>0$ such that $u=0$ a.e. on $[0, h]$. For each integer $n \geq 1$, let us choose $\rho_{n}: \mathbb{R} \rightarrow \mathbb{R}$ to be a mollifier function such that

$$
\operatorname{supp} \rho_{n} \subseteq[0, h], \quad \forall n \geq 1,
$$

where $\operatorname{supp} \rho_{n}$ denotes the support of $\rho_{n}$. Let

$$
\widetilde{u}:=\left\{\begin{array}{ll}
u & \text { on }[0, T] \\
0 & \text { elsewhere }
\end{array},\right.
$$

and define

$$
u_{n}:=\rho_{n} * \widetilde{u} \in L^{p}(\mathbb{R}, X) .
$$

We observe that $u_{n} \in C^{\infty}(\mathbb{R} ; X)$ and $u_{n}(0)=0$ by $(3.6)$. Firstly, it is well known that the restriction of $u_{n}$ converges towards $u$ in $L^{p}(0, T ; X)$. Secondly, let us show that

$$
\mathrm{D}_{0, t}^{\alpha}\left(u_{\left.n\right|_{[0, T]}}\right) \rightarrow \mathrm{D}_{0, t}^{\alpha} u \quad \text { in } L^{q}(0, T ; Y) .
$$

For, let

For each $n \geq 1$, the function

$$
F:=\left\{\begin{array}{ll}
\mathrm{D}_{0, t}^{\alpha} u & \text { on }[0, T] \\
0 & \text { elsewhere }
\end{array} .\right.
$$

$$
v_{n}:=\rho_{n} * g_{\alpha} * F
$$

lies in $L_{\text {loc }}^{1}(\mathbb{R} ; Y)$ and satisfies, according to [LL18a, Lemma 2.2] or [GS77, Chap I.5],

$$
\begin{aligned}
g_{1-\alpha} * v_{n} & =g_{1-\alpha} * \rho_{n} * g_{\alpha} * F \\
& =g_{1-\alpha} * g_{\alpha} * \rho_{n} * F \\
& =g_{1} * \rho_{n} * F .
\end{aligned}
$$

Recalling that $g_{1}$ is the causal function equal to 1 a.e. on $[0, \infty)$, we get by differentiability

$$
\frac{\mathrm{d}}{\mathrm{d} t}\left\{g_{1-\alpha} * v_{n}\right\}=\rho_{n} * F \quad \text { in } \quad L^{q}(\mathbb{R} ; Y)
$$

Whence, since $F=\mathrm{D}_{0, t}^{\alpha} u$ on $[0, T]$,

$$
\mathrm{D}_{0, t}^{\alpha}\left(v_{\left.n\right|_{[0, T]}}\right)=\frac{\mathrm{d}}{\mathrm{d} t}\left\{g_{1-\alpha} * v_{n}\right\}_{\left.\right|_{[0, T]}} \underset{n \rightarrow \infty}{\longrightarrow} \mathrm{D}_{0, t}^{\alpha} u \quad \text { in } L^{q}(0, T ; Y) .
$$

Besides, since $g_{\alpha} * F$ is supported in $[0, \infty[$, one has for a.e. $t \in[0, T]$,

$$
v_{n}(t)=\int_{0}^{t} \rho_{n}(t-y)\left(g_{\alpha} * F\right)(y) \mathrm{d} y
$$


and, by definition of $F$,

$$
\left(g_{\alpha} * F\right)_{[0, T]}=g_{\alpha} * \mathrm{D}_{0, t}^{\alpha} u=u \quad \text { in } L^{q}(0, T ; Y),
$$

thanks to Proposition 2.2 and the fact that $\left(g_{1-\alpha} * u\right)(0)=0$, since $u=0$ a.e. on $[0, h]$. Thus, in view of 3.8 ,

$$
v_{\left.n\right|_{[0, T]}}=u_{\left.n\right|_{[0, T]}} ;
$$

so that 3.9 follows from 3.12 . That completes the proof of the theorem.

Let us now turn to time fractional inequalities. For, let $X$ be a real Banach space densely and continuously embedded into a real Hilbert space $H$. Then $X$ is a Banach subspace of its dual space $X^{\prime}$ and

$$
\langle v, \cdot\rangle_{X^{\prime}, X}=(v, \cdot)_{H}, \quad \forall v \in X
$$

where the bracket denotes the duality between $X^{\prime}$ and $X$, and $(\cdot, \cdot)_{H}$ the inner product of $H$. By [LL18b, Proposition 2.18], any $u$ in $W^{1,1}(0, T ; H)$ with $u(0)=0$, satisfies

$$
\frac{1}{2} g_{1-\alpha} *\|u(\cdot)\|_{H}^{2}(t) \leq \int_{0}^{t}\left(\mathrm{D}_{0, t}^{\alpha} u(s), u(s)\right)_{H} \mathrm{~d} s, \quad \forall t \in[0, T]
$$

Then combining Theorem 3.1 and (3.13), we get easily the following result.

Corollary 3.3. For $X, H$ as above, let $p \geq 2$ whose conjugate exponent is denoted by $p^{\prime}$. Assume that

Then, for each $t \in[0, T]$,

$$
u \in{ }_{0} W_{p, p^{\prime}}^{\alpha}\left(0, T ; X, X^{\prime}\right)
$$

$$
\frac{1}{2} g_{1-\alpha} *\|u(\cdot)\|_{H}^{2}(t) \leq \int_{0}^{t}\left\langle\mathrm{D}_{0, t}^{\alpha} u(s), u(s)\right\rangle_{X^{\prime}, X} \mathrm{~d} s .
$$

That corollary will be useful to get uniqueness results. Regarding existence, the following proposition will be used.

Proposition 3.4. Let $X, H$ as above, $\alpha \in(0,1)$ and $p \geq 2$ be such that $\alpha>1 / p^{\prime}$. Assume

$$
u \in W_{p, p^{\prime}}^{\alpha}\left(0, T ; X, X^{\prime}\right),
$$

and $\left(g_{1-\alpha} * u\right)(0) \in X$. Then

$$
\int_{0}^{T}\left\langle\mathrm{D}_{0, t}^{\alpha} u(t), u(t)-\left(g_{1-\alpha} * u\right)(0) g_{\alpha}(t)\right\rangle_{X^{\prime}, X} \mathrm{~d} t \geq 0 .
$$

Proof. Set for simplicity $v:=\left(g_{1-\alpha} * u\right)(0)$. Since $\alpha>1 / p^{\prime}$, the function $u-v g_{\alpha}$ belongs to $L^{p}(0, T ; X)$. Moreover, for each $t \in[0, T]$, there holds

$$
g_{1-\alpha} *\left(u-v g_{\alpha}\right)(t)=g_{1-\alpha} * u(t)-v \underset{t \rightarrow 0^{+}}{\longrightarrow} 0, \quad \text { in } X^{\prime} .
$$

Hence, $u(t)-v g_{\alpha}$ lies in ${ }_{0} W_{p, p^{\prime}}^{\alpha}\left(0, T ; X, X^{\prime}\right)$ and $\mathrm{D}_{0, t}^{\alpha}\left(u-v g_{\alpha}\right)=\mathrm{D}_{0, t}^{\alpha} u$. Then the assertion follows from Corollary 3.3 .

The following compactness result is proved in [LL18b] for Caputo's derivatives. We just adapt their proof to our framework.

Corollary 3.5. Let $X \subset X_{0} \subset Y$ be Banach spaces such that $X$ is compactly embedded into $X_{0}$. Let $\alpha \in(0,1)$ and $p>1$. Then $W_{p, 1}^{\alpha}(0, T ; X, Y)$ is compactly embedded into $L^{r}\left(0, T ; X_{0}\right)$, for all $r \in[1, p)$. 
Proof. Let $B(0, R)$ denote the closed ball of $W_{p, 1}^{\alpha}(0, T ; X, Y)$ with radius $R>0$ and center 0 . Since $B(0, R)$ is bounded in $L^{p}(0, T ; X)$, it is enough to prove, according to classical Simon's result [Sim87, Theorem 6], that for each $\tau \in(0, T)$ and $h \in[0, T-\tau]$,

$$
\sup _{u \in B(0, R)}\|u(\cdot+h)-u(\cdot)\|_{L^{1}(0, \tau ; Y)} \underset{h \rightarrow 0}{\longrightarrow} 0
$$

For, by Proposition 2.2, we have

$$
u=g_{\alpha}(\cdot) g_{1-\alpha} * u(0)+g_{\alpha} * \mathrm{D}^{\alpha} u \quad \text { in } L^{1}(0, T ; Y) .
$$

Thus, for all $h \in[0, T-\tau]$ such that $h \leq 1$, one has, for a.e. $t \in[0, \tau]$,

$$
\begin{aligned}
& u(t+h)-u(t)=\left(g_{\alpha}(t+h)-g_{\alpha}(t)\right) g_{1-\alpha} * u(0) \\
& \quad+\int_{0}^{t}\left(g_{\alpha}(t+h-y)-g_{\alpha}(t-y)\right) \mathrm{D}^{\alpha} u(y) \mathrm{d} y \\
& \quad+\int_{t}^{t+h} g_{\alpha}(t+h-y) \mathrm{D}^{\alpha} u(y) \mathrm{d} y .
\end{aligned}
$$

Let us estimate the first term in the right hand side of the above equation. Since $W^{1,1}(0, T ; X, Y)$ is embedded into $C([0, T], Y)$, we have

$$
\left\|g_{1-\alpha} * u(0)\right\|_{C([0, T], Y)}^{2} \leq C\left\|g_{1-\alpha} * u\right\|_{L^{1}(0, T, X)}^{2}+C\left\|\mathrm{D}^{\alpha} u\right\|_{L^{1}(0, T, Y)}^{2} .
$$

With (2.1) and $u \in B(0, R)$, we get

$$
\left\|g_{1-\alpha} * u(0)\right\|_{C([0, T], Y)} \leq C\left(\left\|g_{1-\alpha}\right\|_{L^{1}(0, T)}^{2}+1\right)^{1 / 2} R
$$

Besides,

$$
\begin{aligned}
\int_{0}^{\tau}\left|g_{\alpha}(t+h)-g_{\alpha}(t)\right| \mathrm{d} t & =g_{\alpha+1}(\tau)-g_{\alpha+1}(\tau+h)+g_{\alpha+1}(h) \\
& \leq g_{\alpha+1}(h)
\end{aligned}
$$

since $g_{\alpha+1}$ is increasing. There result that the first term is bounded in $L^{1}(0, \tau, Y)$ by $C(R) g_{\alpha+1}(h)$, for some constant $C(R)$ independent of $u$ and $h$.

Regarding the second term, its $L^{1}(0, \tau, Y)$-norm is bounded by

$$
\begin{aligned}
\int_{0}^{\tau} \int_{0}^{t}\left|g_{\alpha}(t+h-y)-g_{\alpha}(t-y)\right| & \left\|\mathrm{D}^{\alpha} u(y)\right\|_{Y} \mathrm{~d} y \mathrm{~d} t \\
\leq & \int_{0}^{\tau}\left\|\mathrm{D}^{\alpha} u(y)\right\|_{Y} \mathrm{~d} y \int_{y}^{\tau} g_{\alpha}(t-y)-g_{\alpha}(t+h-y) \mathrm{d} t,
\end{aligned}
$$

by Fubini's Theorem. Moreover,

$$
\begin{aligned}
\int_{y}^{\tau} g_{\alpha}(t-y)-g_{\alpha}(t+h-y) \mathrm{d} t & =g_{\alpha+1}(\tau-y)-g_{\alpha+1}(\tau+h-y)+g_{\alpha+1}(h) \\
& \leq g_{\alpha+1}(h),
\end{aligned}
$$

since $g_{\alpha+1}$ is increasing. Thus second term is bounded in $L^{1}(0, \tau, Y)$ by $R g_{\alpha+1}(h)$.

We proceed in the same way for the third term of (3.18). Its $L^{1}(0, \tau, Y)$-norm is bounded by

$$
\int_{0}^{\tau+h}\left\|\mathrm{D}^{\alpha} u(y)\right\|_{Y} \mathrm{~d} y \int_{y-h}^{y} g_{\alpha}(t+h-y) \mathrm{d} t \leq R g_{\alpha+1}(h) .
$$

Finally, 3.17 holds which completes the proof of the theorem. 


\section{Time FRACTIONAL SEMILINEAR HEAT EQUATIONS}

Let $n$ be a positive integer, $\Omega$ be a bounded open subset of $\mathbb{R}^{n}, T>0$, and $0<\alpha<1$. The problem under consideration is

$$
\begin{cases}\text { Find } u:[0, T] \times \Omega \rightarrow \mathbb{R} \text { such that } & \\ \mathrm{D}_{0, t}^{\alpha} u-\Delta u+f(u)=0 & \text { on }[0, T] \times \Omega \\ u=0 & \text { on }[0, T] \times \partial \Omega \\ \left(g_{1-\alpha} * u\right)(0, \cdot)=v & \text { on } \partial \Omega .\end{cases}
$$

Here $v: \Omega \rightarrow \mathbb{R}$ is the initial condition and $f: \mathbb{R} \rightarrow \mathbb{R}$ is a non-linear function with polynomial growth at infinity. We will assume that $f$ has the convenient sign at $\pm \infty$ in order to avoid blow-up phenomena and get global in time solutions. These assumptions on $f$ are standard in pattern formation equations (see [Tem88]).

We will solve (4.1) by the Galerkin method. In the standard case where $\alpha=1$, that method consists in solving first a finite dimensional approximated problem, and then pass to the limit. In the fractional case, it turns out that some extra condition is needed for the solvability of the approximated problem. Roughly speaking, that condition looks like a growth condition on the derivative of $f$ (see [ue]). However, it is not needed to pass to the limit. Also such assumption is not needed in the case $\alpha=1$.

In order to avoid that extra assumption, we truncate the non-linear term $f$. Then we solve the truncated fractional PDE by projecting onto a finite dimensional space. Finally, we pass to the limit in the truncated problem. Hence, we will first solve (4.1) for sub-linear $f$. The general case will be investigated in the second subsection.

4.1. The hilbertian case. When $f: \mathbb{R} \rightarrow \mathbb{R}$ has a sub-linear growth, we may work with Hilbert spaces. Thus we have only to control the fractional derivative. More precisely, we will assume that there exists a positive constant $C$ such that

$$
\begin{array}{ll}
|f(u)-f(v)| \leq C|u-v| & \\
f(u) u \geq-C, & \forall u, v \in \mathbb{R} .
\end{array}
$$

Recalling the notation (3.1) for fractional spaces, the problem under consideration is then

$$
\begin{cases}\text { Find } u \in W_{2,2}^{\alpha}\left(0, T ; H_{0}^{1}(\Omega), H^{-1}(\Omega)\right) & \text { such that } \\ \mathrm{D}_{0, t}^{\alpha} u-\Delta u+f(u)=0 & \text { in } L^{2}\left(0, T ; H^{-1}(\Omega)\right) \\ \left(g_{1-\alpha} * u\right)(0)=v & \text { in } L^{2}(\Omega) .\end{cases}
$$

Theorem 4.1. Assume $v \in H_{0}^{1}(\Omega)$ and $f$ satisfies (4.2), (4.3).

(i) If $\alpha \in\left(\frac{1}{2}, 1\right)$ then 4.2 has a unique solution.

(ii) If $\alpha \in\left(0, \frac{1}{2}\right]$ then

(a) if $v \neq 0$ then (4.2) has no solution;

(b) if $v=0$ then (4.2) has a unique solution.

Proof. By Proposition 2.2, we derive that (4.4) has no solution if $\alpha \leq 1 / 2$ and $v \neq 0$. On the other hand, if $v=0$ then the solvability of (4.4) can be achieved as in the case $\alpha \in\left(\frac{1}{2}, 1\right)$. Thus we will only consider in the sequel the case where $\alpha>1 / 2$.

Existence of a solution. We will implement the Galerkin approximation method. For, let us introduce some notation. Denote by $(\cdot, \cdot)_{0}$ the inner product of $L^{2}(\Omega)$ and

$$
A: H_{0}^{1}(\Omega) \rightarrow H^{-1}(\Omega), \quad u \mapsto-\Delta u
$$


For $k=1,2, \ldots$, let $\left(w_{k}, \lambda_{k}\right) \in H_{0}^{1}(\Omega) \times(0, \infty)$ be a $k^{\text {th }}$ mode of $A$ such that $\left(w_{k}\right)_{k \geq 1}$ forms an hilbertian basis of $L^{2}(\Omega)$. For $n=1,2, \ldots$, we denote by $F_{n}$ the vector space generated by $w_{1}, \ldots, w_{n}$. Finally, we decompose the initial condition $v$, by writing

$$
v=\sum_{k \geq 1} b_{k} w_{k} \quad \text { in } H_{0}^{1}(\Omega)
$$

and we set

$$
v_{n}:=\sum_{k=1}^{n} b_{k} w_{k} .
$$

Whence $v_{n} \in F_{n}$ and $v_{n} \rightarrow v$ in $H_{0}^{1}(\Omega)$.

(i) An approximated problem. For each integer $n \geq 1$, our approximated problem takes the form

$$
\left\{\begin{array}{l}
\text { Find } u_{n} \in L^{2}\left(0, T ; F_{n}\right) \text { such that } \mathrm{D}_{0, t}^{\alpha} u_{n} \in L^{2}\left(0, T, F_{n}\right) \\
\left(\mathrm{D}_{0, t}^{\alpha} u_{n}, w\right)_{0}+\left(\nabla u_{n}, \nabla w\right)_{0}+\left(f\left(u_{n}\right), w\right)_{0}=0 \quad \text { in } L^{2}(0, T), \forall w \in F_{n} \\
\left(g_{1-\alpha} * u_{n}\right)(0)=v_{n} .
\end{array}\right.
$$

Thanks (4.2), we may show by standard methods in fractional calculus (see Oue for details) that the ordinary fractional differential equation (4.6) is uniquely solvable.

(ii) Estimates. Since $\alpha>1 / 2$ and $v_{n} \in H_{0}^{1}(\Omega), w:=u_{n}-g_{\alpha} v_{n}$ is a suitable test-function for (4.6), hence

$$
\begin{aligned}
\left(\mathrm{D}_{0, t}^{\alpha} u_{n}, u_{n}-g_{\alpha} v_{n}\right)_{0}+\int_{\Omega}\left|\nabla u_{n}\right|^{2} \mathrm{~d} x+\int_{\Omega} f\left(u_{n}\right) & u_{n} \mathrm{~d} x \\
& =g_{\alpha} \int_{\Omega} \nabla u_{n} \nabla v_{n} \mathrm{~d} x+\int_{\Omega} f\left(u_{n}\right) g_{\alpha} v_{n} \mathrm{~d} x,
\end{aligned}
$$

in $L^{2}(0, T)$. Since $u_{n}$ belongs to $W_{2,2}^{\alpha}\left(0, T ; L^{2}(\Omega), L^{2}(\Omega)\right)$, Proposition 3.4 yields that the time integral of the term involving the fractional derivative is non negative. Then, using (4.2), (4.3) and the boundedness of $\left(v_{n}\right)$ in $H_{0}^{1}(\Omega)$, we derive by standard estimates

$$
\begin{aligned}
& \left\|u_{n}\right\|_{L^{2}\left(0, T ; H_{0}^{1}(\Omega)\right)} \leq C \\
& \left\|\mathrm{D}_{0, t}^{\alpha} u_{n}(t)\right\|_{L^{2}\left(0, T ; H^{-1}(\Omega)\right)} \leq C \\
& \left\|f\left(u_{n}\right)\right\|_{L^{2}\left(0, T ; L^{2}(\Omega)\right)} \leq C .
\end{aligned}
$$

(iii) Passage to the limit. According to (4.7), there exists $u \in L^{2}\left(0, T ; H_{0}^{1}(\Omega)\right)$ such that up to a subsequence

$$
u_{n} \rightarrow u \quad \text { in } L^{2}\left(0, T ; H_{0}^{1}(\Omega)\right) \text {-weak. }
$$

Moreover, by Corollary 3.5. $W_{2,1}^{\alpha}\left(0, T ; H_{0}^{1}(\Omega), H^{-1}(\Omega)\right)$ is compactly embedded into to $L^{1}\left(0, T ; L^{2}(\Omega)\right)$. Thus, up to a subsequence,

$$
u_{n} \rightarrow u \quad \text { a.e on }[0, T] \times \Omega .
$$

Then, by continuity of $f$,

$$
f\left(u_{n}\right) \rightarrow f(u) \quad \text { a.e on }[0, T] \times \Omega .
$$

Thus, using also (4.9), Lion's Lemma [Lio69, lemma I-1.3] yields

$$
f\left(u_{n}\right) \rightarrow f(u) \quad \text { in } L^{2}\left(0, T ; L^{2}(\Omega)\right) .
$$


(iv) Solvability of the equation of (4.4). Let us show that

$$
\mathrm{D}_{0, t}^{\alpha} u-\Delta u+f(u)=0 \quad \text { in } L^{2}\left(0, T ; H^{-1}(\Omega)\right) .
$$

For, let $k \geq 1$ be fixed and $n \geq k$. For each $\varphi \in \mathcal{D}(0, T)$, we derive from 4.6), Proposition 2.4 and Proposition 2.3 that

$$
\left\langle\int_{0}^{T}-u_{n}(t) \mathrm{D}_{t, T}^{\alpha} \varphi(t)+\left(A u_{n}-f\left(u_{n}\right)\right) \varphi(t) \mathrm{d} t, w_{k}\right\rangle_{H^{-1}(\Omega), H_{0}^{1}(\Omega)}=0 .
$$

Passing to the limit in $n$ and using Definition 2.4, we get

$$
\mathbf{D}^{\alpha} u+A u+f(u)=0 \quad \text { in } \mathcal{D}^{\prime}\left(0, T ; H^{-1}(\Omega)\right) .
$$

Since $A u$ and $f(u)$ belong to $L^{2}\left(0, T ; H^{-1}(\Omega)\right)$, we derive that $u$ lies in $W_{2,2}^{\alpha}\left(0, T ; H_{0}^{1}(\Omega), H^{-1}(\Omega)\right)$ and

$$
\mathbf{D}^{\alpha} u+A u+f(u)=0 \quad \text { in } L^{2}\left(0, T ; H^{-1}(\Omega)\right) .
$$

(v) Initial condition. Let $1 \leq k \leq n$ and $\psi \in H^{1}(0, T)$ with $\psi(T)=0$. Then, starting from (4.10) and using Proposition 2.4 and Proposition 2.3, we derive

$$
\begin{aligned}
-\int_{0}^{T}\left(u(t), w_{k}\right)_{0} \mathrm{D}_{t, T}^{\alpha} \psi(t) \mathrm{d} t-\left(\left(g_{1-\alpha} * u\right)(0), w_{k}\right)_{0} \psi(0) \\
+\int_{0}^{T}\left\langle A u, w_{k}\right\rangle_{H^{-1}(\Omega), H_{0}^{1}(\Omega)} \psi(t) \mathrm{d} t+\int_{0}^{T}\left(f(u(t)), w_{k}\right)_{0} \psi(t) \mathrm{d} t=0
\end{aligned}
$$

Besides, going back to the equation of (4.6) and proceeding in the same way, we get

$$
\begin{aligned}
-\int_{0}^{T}\left(u_{n}(t), w_{k}\right)_{0} \mathrm{D}_{t, T}^{\alpha} \psi(t) \mathrm{d} t-\left(\left(g_{1-\alpha} * u_{n}\right)(0), w_{k}\right)_{0} \psi(0) \\
+\int_{0}^{T}\left\langle A u_{n}, w_{k}\right\rangle_{H^{-1}(\Omega), H_{0}^{1}(\Omega)} \psi(t) \mathrm{d} t+\int_{0}^{T}\left(f\left(u_{n}(t)\right), w_{k}\right)_{0} \psi(t) \mathrm{d} t=0 .
\end{aligned}
$$

We pass to the limit to get

$$
\begin{aligned}
-\int_{0}^{T}\left(u(t), w_{k}\right)_{0} \mathrm{D}_{t, T}^{\alpha} \psi(t) \mathrm{d} t-\left(v, w_{k}\right)_{0} \psi(0) \\
+\int_{0}^{T}\left\langle A u, w_{k}\right\rangle_{H^{-1}(\Omega), H_{0}^{1}(\Omega)} \psi(t) \mathrm{d} t+\int_{0}^{T}\left(f(u(t)), w_{k}\right)_{0} \psi(t) \mathrm{d} t=0 .
\end{aligned}
$$

Comparing 4.11) with 4.12, we deduce that $\left(g_{1-\alpha} * u\right)(0)=v$.

Uniqueness for Problem (4.4). Let $u_{1}, u_{2}$ be two solutions to (4.4). Then $u:=u_{1}-u_{2}$ satisfies

$$
\begin{aligned}
& \mathrm{D}_{0, t}^{\alpha} u-\Delta u+f\left(u_{1}\right)-f\left(u_{2}\right)=0 \quad \text { in } \quad L^{2}\left(0, T ; H^{-1}(\Omega)\right) \\
& \left(g_{1-\alpha} * u\right)(0)=0 .
\end{aligned}
$$

Let $\tau \in(0, T]$. Testing 4.13 with $u$, we derive with the Lipschitz assumption 4.2

$$
\int_{0}^{\tau}\left\langle\mathrm{D}_{0, t}^{\alpha} u(t), u(t)\right\rangle_{H^{-1}(\Omega), H_{0}^{1}(\Omega)} \mathrm{d} t \leq C \int_{0}^{\tau}\|u(t)\|_{L^{2}(\Omega)}^{2} \mathrm{~d} t .
$$


By (4.14), $u$ lies in ${ }_{0} W_{2,2}^{\alpha}\left(0, T ; H_{0}^{1}(\Omega), H^{-1}(\Omega)\right)$. Then Corollary 3.3 yields

$$
g_{1-\alpha} *\|u(.)\|_{L^{2}(\Omega)}^{2}(\tau) \leq 2 C \int_{0}^{\tau}\|u(t)\|_{L^{2}(\Omega)}^{2} d t .
$$

If there exists $\tau \in(0, T]$ such that $u=0$ a.e. on $[0, \tau]$ then we set

$$
t_{0}:=\sup \{\tau \in(0, T]: u=0 \text { a.e. on }[0, \tau]\} \text {. }
$$

Otherwise, we put $t_{0}:=0$. Now, in order to get uniqueness, it is enough to show that $t_{0}=T$. Arguing by contradiction, let us assume that $t_{0} \in[0, T)$. Then for each $\tau \in\left(t_{0}, T\right]$, we have

$$
\int_{t_{0}}^{\tau}\|u(t)\|_{L^{2}(\Omega)}^{2} \mathrm{~d} t=\int_{0}^{\tau}\|u(t)\|_{L^{2}(\Omega)}^{2} \mathrm{~d} t \neq 0 .
$$

Then going back to (4.16) and using the decay of $g_{1-\alpha}$, we derive

$$
g_{1-\alpha}\left(\tau-t_{0}\right) \int_{t_{0}}^{\tau}\|u(t)\|_{L^{2}(\Omega)}^{2} \mathrm{~d} t \leq 2 L \int_{t_{0}}^{\tau}\|u(t)\|_{L^{2}(\Omega)}^{2} d t .
$$

The condition $\int_{t_{0}}^{t}\|u(y)\|_{L^{2}(\Omega)}^{2} \mathrm{~d} y \neq 0$ leads to the boundedness of $\tau \mapsto g_{1-\alpha}\left(\tau-t_{0}\right)$ on $\left(t_{0}, T\right]$. That impossibility shows that $t_{0}=T$. The proof of the theorem is now completed.

4.2. The polynomial growth case. We will assume that the reaction term $f$ has a polynomial growth at infinity. Thus we cannot work no more with fractional Hilbert spaces. However, our functional framework remains, in some sense hilbertian, since the initial condition is constrained to stay in a subspace of $L^{2}(\Omega)$.

Let $n$ be a positive integer, $\Omega$ be a bounded open subset of $\mathbb{R}^{n}$, and $0<\alpha<1$. Let $f: \mathbb{R} \rightarrow \mathbb{R}$ satisfy, for some positive constants $C, c$ and $p$

$$
\begin{aligned}
& f(u) u \geq c|u|^{p+1}-C \\
& |f(u)| \leq C|u|^{p}+C, \quad \forall u \in \mathbb{R} \\
& f \text { is non decreasing on some neighborhood of }-\infty \text { and } \infty \\
& f \in W_{\text {loc }}^{1,1}(\mathbb{R}) .
\end{aligned}
$$

The latter condition means that $f$ is a locally Lipschitz function on $\mathbb{R}$. Also, 4.19 means that there exists some $M_{0} \in(0, \infty)$ such that $f$ is non decreasing on $\left(-\infty,-M_{0}\right]$ and on $\left[M_{0}, \infty\right)$.

Let us denote by $(p+1)^{\prime}$ the conjugate exponent of $p+1$ i.e. $(p+1)^{\prime} \in(1, \infty)$ and

$$
(p+1)^{\prime}:=\frac{p+1}{p} \Longleftrightarrow \frac{1}{(p+1)^{\prime}}+\frac{1}{p+1}=1 \text {. }
$$

The problem under consideration is

$$
\left\{\begin{array}{l}
\text { Find } u \in L^{2}\left(0, T ; H_{0}^{1}(\Omega)\right) \cap L^{p+1}\left(0, T ; L^{p+1}(\Omega)\right) \quad \text { such that } \\
\mathrm{D}_{0, t}^{\alpha} u \in L^{2}\left(0, T ; H^{-1}(\Omega)\right)+L^{(p+1)^{\prime}}\left(0, T ; L^{(p+1)^{\prime}}(\Omega)\right) \\
\mathrm{D}_{0, t}^{\alpha} u-\Delta u+f(u)=0 \quad \text { in } L^{2}\left(0, T ; H^{-1}(\Omega)\right)+L^{(p+1)^{\prime}}\left(0, T ; L^{(p+1)^{\prime}}(\Omega)\right) \\
\left(g_{1-\alpha} * u\right)(0)=v .
\end{array}\right.
$$

Let us notice that 4.21 is a natural extension of the standard case where $\alpha=1$. See for instance [Tem88], [Lio69] or [Chi00].

Moreover, for each

$$
u \in L^{p+1}\left(0, T ; L^{p+1}(\Omega)\right),
$$


(4.18) and the Hölder inequality yield that

$$
f(u) \in L^{(p+1)^{\prime}}\left(0, T ; L^{(p+1)^{\prime}}(\Omega)\right) .
$$

Hence the three terms involved in the equation of Problem 4.21 belong to

$$
L^{2}\left(0, T ; H^{-1}(\Omega)\right)+L^{(p+1)^{\prime}}\left(0, T ; L^{(p+1)^{\prime}}(\Omega)\right) .
$$

If $p \geq 1$ and

$$
u \in L^{2}\left(0, T ; H_{0}^{1}(\Omega)\right), \quad \mathrm{D}_{0, t}^{\alpha} u \in L^{2}\left(0, T ; H^{-1}(\Omega)\right)+L^{(p+1)^{\prime}}\left(0, T ; L^{(p+1)^{\prime}}(\Omega)\right)
$$

then $u$ has a fractional derivative in $L^{(p+1)^{\prime}}\left(0, T ; H^{-1}(\Omega)+L^{(p+1)^{\prime}}(\Omega)\right)$. Hence $g_{1-\alpha} * u$ lies in $C\left([0, T] ; H^{-1}(\Omega)+L^{(p+1)^{\prime}}(\Omega)\right)$. Therefore the initial condition in Problem (4.21) is meaning full.

Now, we may state our main result.

Theorem 4.2. Let us assume the following.

(i) $\alpha \in(0,1), p \in[1, \infty)$;

(ii) $v \in H_{0}^{1}(\Omega) \cap L^{p+1}(\Omega)$;

(iii) $f$ satisfies 4.17 - 4.20 .

Then

(a) if $\alpha>\frac{p}{p+1}$ then 4.21 has a unique solution;

(b) if $\alpha \leq \frac{1}{p+1}$ then

(b-1) if $v \neq 0$ then 4.21 has no solution;

(b-2) if $v=0$ then (4.21) has a unique solution.

For sake of simplicity, we set

$$
V_{p}:=H_{0}^{1}(\Omega) \cap L^{p+1}(\Omega),
$$

and denote its dual space by $V_{p}^{\prime}$.

Proof of Theorem 4.2. Let $\alpha \leq \frac{1}{p+1}$ and $u$ be a solution to 4.21. Since $p \geq 1$, we have $(p+1)^{\prime} \leq 2$. Thus $u$ and $\mathrm{D}^{\alpha} u$ belong to $L^{(p+1)^{\prime}}\left(0, T ; V_{p}^{\prime}\right)$. Hence Proposition 2.2 yields

$$
\left(g_{1-\alpha} * u\right)(0) g_{\alpha} \in L^{(p+1)^{\prime}}\left(0, T ; V_{p}^{\prime}\right) .
$$

Since $g_{\alpha} \notin L^{(p+1)^{\prime}}(0, T)$, the initial condition $v$ must be trivial. In that case, existence and uniqueness may be achieved as in the case $\alpha>\frac{p}{p+1}$. So, in the sequel of that proof, we will assume that $\alpha>\frac{p}{p+1}$.

Existence of a solution. (i) A truncated problem. For all positive integer $M$, we define $f_{M}: \mathbb{R} \rightarrow \mathbb{R}$ by

$$
f_{M}(u)= \begin{cases}f(u) & \text { if }|u| \leq M \\ f(M) & \text { if } u>M \\ f(-M) & \text { if } u<-M\end{cases}
$$

By 4.17, one has

$$
f_{M}(u) u \geq-C, \quad \forall u \in \mathbb{R}
$$


where $C$ is the constant appearing in 4.17). Then, according to Theorem 4.1, the following truncated problem

$$
\begin{cases}\text { Find } u_{M} \in W_{2,2}^{\alpha}\left(0, T ; H_{0}^{1}(\Omega), H^{-1}(\Omega)\right) & \text { such that } \\ \mathrm{D}_{0, t}^{\alpha} u_{M}-\Delta u_{M}+f_{M}\left(u_{M}\right)=0 & \text { in } L^{2}\left(0, T ; H^{-1}(\Omega)\right) \\ \left(g_{1-\alpha} * u_{M}\right)(0)=v & \text { in } L^{2}(\Omega) .\end{cases}
$$

has a unique solution since $\alpha>\frac{p}{p+1} \geq 1 / 2$. Observe that $f_{M}$ converges toward $f$ uniformly on compact sets of $\mathbb{R}$. Thus it is expected that, in the limit $M \rightarrow \infty, u_{M}$ gives a solution to 4.21.

(ii) Estimates. Arguing as in the proof of Theorem 4.1, we get, using in particular Proposition 3.4

$$
\int_{0}^{T} \int_{\Omega}\left|\nabla u_{M}\right|^{2} \mathrm{~d} x+f_{M}\left(u_{M}\right) u_{M} \mathrm{~d} x \mathrm{~d} t \leq C+C \int_{0}^{T} \int_{\Omega} f_{M}\left(u_{M}\right) g_{\alpha} v \mathrm{~d} x \mathrm{~d} t .
$$

Young inequality and Lemma 4.3 below lead to

$$
\begin{aligned}
\left|f_{M}\left(u_{M}\right) g_{\alpha} v\right| & \leq \varepsilon\left|f_{M}\left(u_{M}\right)\right|^{(p+1)^{\prime}}+C_{\varepsilon}\left|g_{\alpha} v\right|^{p+1} \\
& \leq \varepsilon C_{0} f_{M}\left(u_{M}\right) u_{M}+\varepsilon C_{0}+C_{\varepsilon}\left|g_{\alpha} v\right|^{p+1}, \quad \forall M \geq M_{0},
\end{aligned}
$$

where $C_{0}$ and $M_{0}$ are the constants appearing in Lemma 4.3 . Thus

$$
\int_{0}^{T} \int_{\Omega}\left|\nabla u_{M}\right|^{2}+f_{M}\left(u_{M}\right) u_{M} \mathrm{~d} x \mathrm{~d} t \leq C .
$$

Also, by 4.27) and Lemma 4.3 again,

$$
\int_{0}^{T} \int_{\Omega}\left|f_{M}\left(u_{M}\right)\right|^{(p+1)^{\prime}} \mathrm{d} x \mathrm{~d} t \leq C .
$$

Let us now show that the sequence $\left(\mathrm{D}^{\alpha} u_{M}\right)_{M \geq 0}$ remains bounded in $L^{1}\left(0, T, V_{p}^{\prime}\right)$. Indeed, testing (4.26) with $w \in V_{p}$, and using Hölder inequality, we arrive to

$$
\left|\left\langle\mathrm{D}^{\alpha} u_{M}, w\right\rangle_{H^{-1}(\Omega)}\right| \leq\left\|u_{M}\right\|_{H_{0}^{1}(\Omega)}\|w\|_{H_{0}^{1}(\Omega)}+\left\|f_{M}\left(u_{M}\right)\right\|_{L^{(p+1)^{\prime}}(\Omega)}\|w\|_{L^{p+1}(\Omega)} .
$$

Moreover, by density, $H^{-1}(\Omega)$ is a subspace of $V_{p}^{\prime}$. Thus, with 4.27) and 4.28

$$
\int_{0}^{T}\left\|\mathrm{D}^{\alpha} u_{M}\right\|_{V_{p}^{\prime}} \mathrm{d} t \leq C, \quad \forall M \geq M_{0}
$$

(iii) Passage to the limit. According to 4.27) and 4.28), Corollary 3.5 yields the existence of some $u \in L^{1}\left(0, T ; L^{2}(\Omega)\right)$ such that up to a subsequence,

$$
u_{M} \underset{M \rightarrow \infty}{\longrightarrow} u \quad \text { a.e. on }[0, T] \times \Omega \text {. }
$$

Since $f_{M}$ converges toward $f$ uniformly on compact sets of $\mathbb{R}$, there holds $f_{M}\left(u_{M}\right) \rightarrow f(u)$ a.e. on $[0, T] \times \Omega$. Thus, using also 4.25) and 4.27), Fatou's Lemma leads to

$$
\int_{0}^{T} \int_{\Omega} f(u) u \mathrm{~d} x \mathrm{~d} t \leq C
$$

Thus, with 4.17), we get that $u \in L^{p+1}\left(0, T ; L^{p+1}(\Omega)\right)$, and (see 4.22$)$ ) that $f(u)$ lies in $L^{(p+1)^{\prime}}\left(0, T ; L^{(p+1)^{\prime}}(\Omega)\right)$. Then Lion's Lemma yields

$$
f_{M}\left(u_{M}\right) \rightarrow f(u) \quad \text { in } L^{(p+1)^{\prime}}\left(0, T ; L^{(p+1)^{\prime}}(\Omega)\right) .
$$


For any $w \in V_{p}$ and $\varphi \in \mathcal{D}(0, T)$, testing 4.26 with $w \varphi$ and using Proposition 2.4, we get in a standard way

$$
\mathrm{D}_{0, t}^{\alpha} u-\Delta u+f(u)=0 \quad \text { in } L^{2}\left(0, T ; H^{-1}(\Omega)\right)+L^{(p+1)^{\prime}}\left(0, T ; L^{(p+1)^{\prime}}(\Omega)\right) .
$$

(v) Initial condition. Since $(p+1)^{\prime} \leq 2$, 4.31) holds in $L^{1}\left(0, T ; V_{p}^{\prime}\right)$. Then testing 4.31 with $w \psi$ for any $w \in V_{p}$ and $\psi \in H^{1}(0, T)$ such that $\psi(T)=0$, we derive, by applying Proposition 2.4 in $L^{1}\left(0, T ; V_{p}^{\prime}\right)$,

$$
\begin{aligned}
& -\int_{0}^{T}\langle u(t), w\rangle_{V_{p}^{\prime}, V_{p}} \mathrm{D}_{t, T}^{\alpha} \psi(t) \mathrm{d} t-\left\langle\left(g_{1-\alpha} * u\right)(0), w\right\rangle_{V_{p}^{\prime}, V_{p}} \psi(0) \\
& \quad+\int_{0}^{T}\langle A u, w\rangle_{H^{-1}(\Omega), H_{0}^{1}(\Omega)} \psi(t) \mathrm{d} t+\int_{0}^{T} \int_{\Omega} f(u(t)) w \psi(t) \mathrm{d} x \mathrm{~d} t=0
\end{aligned}
$$

On the other hand arguing as in the proof of Theorem 4.1, we obtain

$$
\begin{aligned}
-\int_{0}^{T}(u(t), w)_{0} \mathrm{D}_{t, T}^{\alpha} \psi(t) \mathrm{d} t-(v, w)_{0} \psi(0) & \\
& \int_{0}^{T}\langle A u, w\rangle_{H^{-1}(\Omega), H_{0}^{1}(\Omega)} \psi(t) \mathrm{d} t+\int_{0}^{T} \int_{\Omega} f(u(t)) w \psi(t) \mathrm{d} x \mathrm{~d} t=0 .
\end{aligned}
$$

Comparing 4.32 with 4.33 , we deduce that $\left(g_{1-\alpha} * u\right)(0)=v$.

Uniqueness for Problem 4.21). We proceed as in the proof of Theorem 4.1. The nonlinear term is controlled in a usual way, by using the following property. There exists $M>0$ such that

$$
(f(u)-f(v))(u-v) \geq 0, \quad \forall|u| \geq M, \forall v \in \mathbb{R} .
$$

See [Oue for details. The proof of the theorem is now completed.

If $p>1$ then the theorem tells nothing when $\frac{1}{p+1}<\alpha \leq \frac{p}{p+1}$. More regularity on $\mathrm{D}_{0, t}^{\alpha} u$ allows to fill that gap. Indeed, let $\alpha \leq \frac{p}{p+1}$ and $u$ be a solution to 4.21 such that

$$
\mathrm{D}_{0, t}^{\alpha} u \in L^{p+1}\left(0, T ; H^{-1}(\Omega)+L^{(p+1)^{\prime}}(\Omega)\right) .
$$

Then, according to the proof of Theorem $4.2, v=0$; so that 4.21 has a unique solution for $v=0$ and $\alpha \leq \frac{p}{p+1}$.

The following result is used in the proof of Theorem 4.2. We refer to Oue for its proof.

Lemma 4.3. Let $f: \mathbb{R} \rightarrow \mathbb{R}$ satisfy 4.17, 4.18 with $p>0$ and $f_{M}$ defined by 4.24. Then there exist $M_{0}>0$ and $C_{0}>0$ such that

$$
|f(u)|^{\frac{p+1}{p}} \leq C_{0} f_{M}(u) u+C_{0}, \quad \forall u \in \mathbb{R}, \quad \forall M \geq M_{0} .
$$

\section{REFERENCES}

[Chi00] Michel Chipot. Elements of nonlinear analysis. Birkhäuser Advanced Texts. Birkhäuser Verlag, Basel, 2000.

[dF19] Paulo M. de Carvalho Neto and Renato Fehlberg Junior. On the fractional version of Leibniz rule. arXiv e-prints, page arXiv:1901.10376, Jan 2019.

[DR18] Laid Djilali and Arnaud Rougirel. Galerkin method for time fractional diffusion equations. $J$. Elliptic Parabol. Equ., 4(2):349-368, 2018.

[GS77] I. M. Gelfand and G. E. Shilov. Generalized functions. Vol. 1. Academic Press, New York-London, 1964 [1977]. Properties and operations. 
[Lio69] J.-L. Lions. Quelques méthodes de résolution des problèmes aux limites non linéaires. Dunod; Gauthier-Villars, Paris, 1969.

[LL18a] Lei Li and Jian-Guo Liu. A generalized definition of Caputo derivatives and its application to fractional ODEs. SIAM J. Math. Anal., 50(3):2867-2900, 2018.

[LL18b] Lei Li and Jian-Guo Liu. Some compactness criteria for weak solutions of time fractional PDEs. SIAM J. Math. Anal., 50(4):3963-3995, 2018.

[Oue] Yamina Ouedjedi. Phd Thesis of the university of Mascara.

[Rou05] Tomas Roubicek. Nonlinear partial differential equations with applications, volume 153 of International Series of Numerical Mathematics. Birkhäuser Verlag, Basel, 2005.

[Sim87] Jacques Simon. Compact sets in the space $L^{p}(0, T ; B)$. Ann. Mat. Pura Appl. (4), 146:65-96, 1987.

[Tem88] Roger Temam. Infinite-dimensional dynamical systems in mechanics and physics, volume 68 of Applied Mathematical Sciences. Springer-Verlag, New York, 1988.

[Zac12] Rico Zacher. Global strong solvability of a quasilinear subdiffusion problem. J. Evol. Equ., 12(4):813-831, 2012.

YAMINA OUEDJEDI,

Department of Mathematics, Faculty of Exact Sciences, University of Mascara

E-mail address: oudjediyamina@gmail.com

ARnaud Rougirel

Laboratoire de Mathématiques, Université de Poitiers \& CNRS. teleport 2, BP 179, 86960

Chassneuil du Poitou Cedex, France,

E-mail address: rougirel@math.univ-poitiers.fr

KHALED BENMERIEM,

Department of Mathematics, Faculty of Exact Sciences, University of Mascara

E-mail address: benmeriem@univ-mascara.dz 